\title{
Identification of genomic functional hotspots with copy number alteration in liver cancer
}

\author{
Tzu-Hung Hsiao ${ }^{1}$, Hung-l Harry Chen ${ }^{1}$, Stephanie Roessler ${ }^{2}$, Xin Wei Wang ${ }^{3}$ and Yidong Chen ${ }^{1,4^{*}}$
}

\begin{abstract}
Copy number alterations (CNAs) can be observed in most of cancer patients. Several oncogenes and tumor suppressor genes with CNAs have been identified in different kinds of tumor. However, the systematic survey of CNA-affected functions is still lack. By employing systems biology approaches, instead of examining individual genes, we directly identified the functional hotspots on human genome. A total of 838 hotspots on human genome with 540 enriched Gene Ontology functions were identified. Seventy-six aCGH array data of hepatocellular carcinoma (HCC) tumors were employed in this study. A total of 150 regions which putatively affected by CNAs and the encoded functions were identified. Our results indicate that two immune related hotspots had copy number alterations in most of patients. In addition, our data implied that these immune-related regions might be involved in HCC oncogenesis. Also, we identified 39 hotspots of which copy number status were associated with patient survival. Our data implied that copy number alterations of the regions may contribute in the dysregulation of the encoded functions. These results further demonstrated that our method enables researchers to survey biological functions of CNAs and to construct regulation hypothesis at pathway and functional levels.
\end{abstract}

Keywords: Copy number alteration; Gene set enrichment; Pathway analysis; Liver cancer

\section{Introduction}

Chromosomal instability is one of the characteristics in cancer [1] and results in the numerical and structural alterations of DNA copy number variations (CNAs). Recently, some literatures have reported the association of CNAs and patient survival in different tumors [2-4]. Several important oncogenes or tumor suppressors were also showed with high frequency of gain or loss status in different cancers. For example, the copy number amplification of gene Her2, which is the addicted oncogene in the HER2+ subtype of breast cancer, was highly correlated with the gene overexpression [5]. However, in addition to focal amplification, most tumors display multiple and broad ranges of copy number change, where large number of genes are involved in and potentially to be induced or suppressed due to copy number amplifications or deletions. Some in vitro studies were performed to survey the affected functions of CNAs [6-8]. For example,

\footnotetext{
* Correspondence: cheny8@uthscsa.edu

${ }^{1}$ Greehey Children's Cancer Research Institute, University of Texas Health

Science Center at San Antonio, San Antonio, TX 78229, USA

${ }^{4}$ Department of Epidemiology and Biostatistics, University of Texas Health

Science Center at San Antonio, San Antonio, TX 78229, USA

Full list of author information is available at the end of the article
}

Nicole et al. utilized the shRNA library to identify the GO and STOP genes which positively and negatively regulate proliferation to evaluate the effect of gene deletions [7], respectively. They also proposed a model called 'Cancer Gene Island', which encompasses high density of genes with the same function within a genomic region [7]. However, the in vitro studies were labor intensive if not cost prohibitive. Moreover, it is hard to perform a systematic analysis based on these approaches, thus, leaving the gene island model and their functions unexplored.

In conventional gene expression data analysis, several bioinformatics methods based on the concept of 'gene set enrichment analysis' (GSEA) have been successfully utilized to explore the underlying molecular pathways and Gene Ontology functions [9-12]. The GSEA method assesses the number of overlap genes between two gene sets: the differentially expressed genes of a certain functional annotation and genes from the entire genome with the same annotation, to estimate the probability of the overlapping through the statistical test. The procedure provides a high throughput and systematic analysis to explore the putative activated pathways or functions.

\section{实}

(c) 2013 Hsiao et al.; licensee Springer. This is an open access article distributed under the terms of the Creative Commons Attribution License (http://creativecommons.org/licenses/by/2.0), which permits unrestricted use, distribution, and reproduction in any medium, provided the original work is properly cited. 
Hepatocellular carcinoma (HCC) is one of the malignant cancers and the third leading cause of cancer death worldwide [13]. Major etiologies associated with HCC are hepatitis $B$ virus (HBV) and hepatitis $\mathrm{C}$ virus (HCV) infection [14]. Previous studies have been reported in which comparative genomic hybridization by microarray $(\mathrm{aCGH})$ was utilized to examine CNAs in HCC. Several regions with frequent copy number gain and loss were identified. The CNA-associated oncogenes and tumor suppressors, such as MYC, JAG1, TP53, and RB1, were also found [15-18]. The association between survival and CNAs has been investigated, and ten associated genes were reported [19]. However, the biological functions altered by CNAs remain unknown and thus need to be dissected.

According to the concept of Cancer Gene Island, here, we propose an algorithm to identify the spatial functional hotspots (SFHs) in human genome based on the enrichment analysis. The human genome is divided firstly into segments along the genomic sequence coordinate. Then, the tests of enrichment between the segments and whole genome functional categories are performed. Finally, a method which identifies the optimal regions of enriched functions between the segments was applied to examine putative SFHs. To demonstrate the ability of our method, we applied the method to an aCGH data set of HCC. The result showed several immune-related SFHs which showed gain and loss in HCC samples. Also, survival-associated SFHs were identified. The result also indicated that our system could serve as a useful method to understand the CNAs-affected functions.

\section{Methods}

To identify the SFHs in human genome, we proposed a novel enrichment analysis that compares the genes contained within a genomic segment with all genes belonging to the same function categories associated to the genes within the segment under consideration based on the concept of gene set enrichment. As shown in Figure 1A, two matrixes, $\mathbf{B}$ and $\mathbf{P}$, were constructed first. The indicator matrix $\mathbf{B}$ contains information whether or not a gene belongs to a genomic region (spatial segment) determined by a sliding window along the genomic position of all chromosomes or $\mathbf{B}=\left(b_{k, i}\right)_{K \times M}$, where $M$ is the number of genes and $K$ is the number of genome segments pre-determined and where $b_{k, i}=1$ when $i$ th gene is in the $k$ th segment, otherwise 0 . The matrix $\mathbf{P}=\left\{p_{i, l}\right\}_{M \times L}$ is also an indicator matrix of functional gene sets, where $L$ is the number of functional gene sets and $p_{i, l}=1$ when $i$ th gene is in the $l$ th GO (Gene Ontology) function, otherwise 0 . The enrichment is defined as scoring function $C$ of the two matrixes $\mathbf{B}$ and $\mathbf{P}$

$$
E S=C(\mathbf{B}, \mathbf{P})
$$

Here, we use Fisher's exact test as the score function $C$ $(\mathbf{B}, \mathbf{P})$ (Figure 1B). Let $x_{k}=\sum_{i=1}^{M} b_{k, i}$ is the number of genes in the $k$ th segment, $y_{l}=\sum_{i=1}^{M} p_{i, l}$ is the number of total genes in gene set $l$, and $z_{k, l}=\sum_{i=1}^{M} b_{k, i} p_{i, l}$ is the number of overlapped genes between the $k$ th segment and gene set $l$. The $p$ value of Fisher's exact test between the genome segment and the gene set can be calculated by

$$
P\left(x>z_{k, l}\right)=\sum_{h=z_{k, l}}^{\infty} \frac{\left(\begin{array}{c}
x_{k} \\
h
\end{array}\right)\left(\begin{array}{c}
M-x_{k} \\
y_{l}-h
\end{array}\right)}{\left(\begin{array}{c}
M \\
y_{l}
\end{array}\right)}
$$

Based on the $p$ values, we can determine if the function $l$ was enriched at the genome segment $k$. Then, we merge and extend the enriched segments to a merged window to include all genes involved in the function $l$ if the segments were located nearby and have position overlapping. As shown in Figure $1 C$, assuming $q$ th to $(q+R)$ th segments have enrichment for the function $l$, the genes involved in the merged windows can be expressed as vector $\boldsymbol{d}$ :

$$
d_{i}=\left\{\begin{array}{cc}
1, & \text { if } s_{i}>1 \\
0, & \text { else }
\end{array},\right.
$$

where $s_{i}=\sum_{t=q}^{(q+R)} b_{t, i}$. Assuming there are $G$ genes (from eth to $(e+G)$ th $)$ located in the $q$ th to $(q+R)$ th enriched window, we defined the subsets of the $G$ genes which exclude out genes gradually from left or right side according to the genome coordinate. Two parameters, $p L$ and $p R$, which perform enrichment analysis (Fisher's exact test) between the subsets with the gene set of function $l$ were introduced (Figure 1C). $p L$ and $p R$ are defined as:

$$
\begin{aligned}
& p R_{g}=C\left(\left\{d_{e+g}, \ldots, d_{(e+G)}\right\}, \mathbf{p}_{l}\right) \\
& p L_{g}=C\left(\left\{d_{e}, \ldots, d_{e+g}\right\}, \mathbf{p}_{l}\right),
\end{aligned}
$$

where $g=1, \ldots, G$ and $C($.$) is the enriched score func-$ tion. Then, the optimal enriched region $o$ of function $l$ can be defined as:

$$
o=\{\operatorname{argmin}(p R), \ldots, \operatorname{argmin}(p L)\} .
$$

If the $p$ value of the region $o$ passed the selection threshold, $o$ was defined as the SFHs of function $l$. 
A
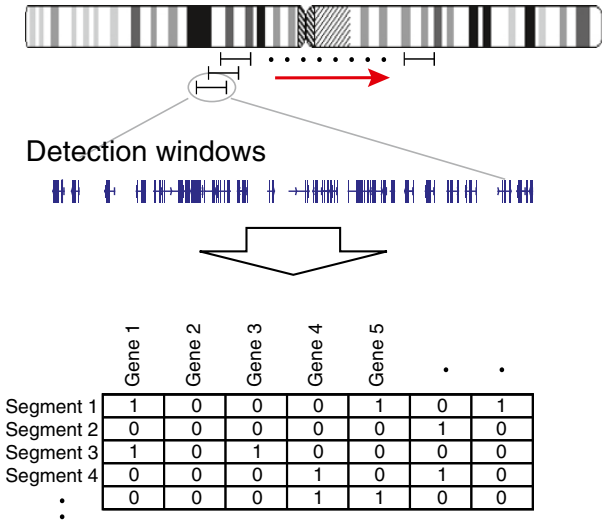

B

B

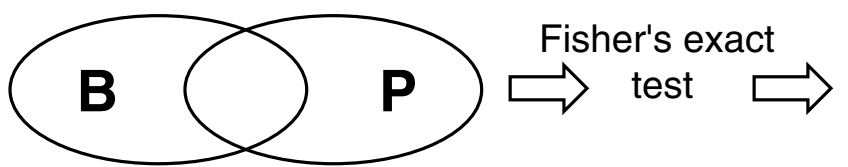
\# of genes in the
\# of genes in genome segment $i$ the geneset $j$

C

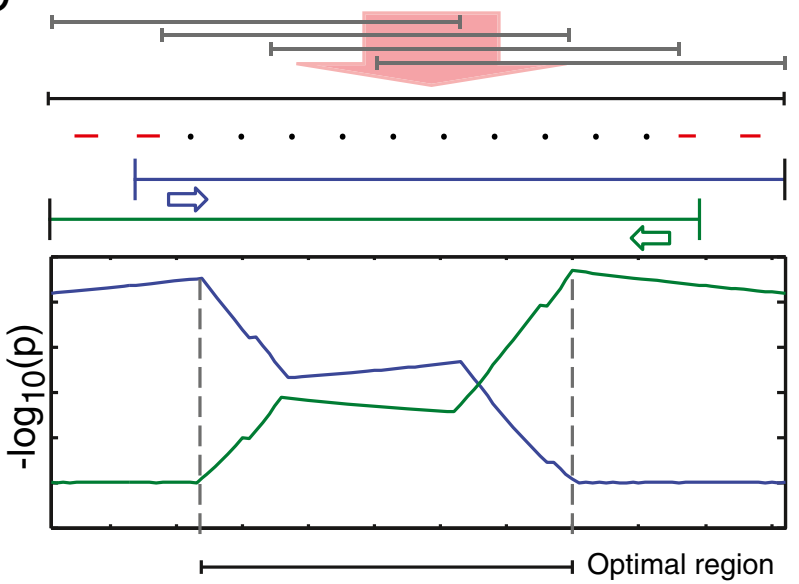

Functional gene set

Gene Ontology

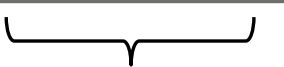

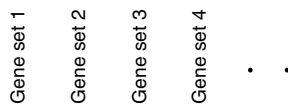

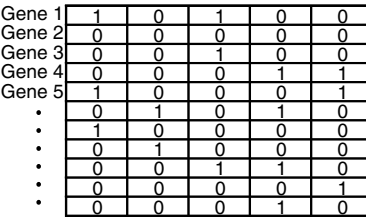

$\mathbf{P}$

p-value

Seg $N$

0.03 Geneset 1

0.20 Geneset 4

\begin{tabular}{ll}
0.06 \\
\hline 0.04 \\
\hline 0.03 \\
\hline
\end{tabular}

0.01

$q^{\text {th }}$ to $(q+R)^{\text {th }}$ enriched seqment

Merged detection window

e to $(e+G)^{\text {th }}$ genes

Right Left side enrichment test

Figure 1 Schematic diagram illustrating the enrichment analysis of spatial functional hotspots in human genome. (A) The indicator matrix $\mathbf{B}$ was generated by sliding the detection window along the genome. It contains the information of genes located in each segment. The matrix $\mathbf{P}$ records the gene sets of Gene Ontology. (B) By comparing the two matrices assessed with Fisher's exact test, the $p$ values of gene sets in each segment were generated. The enriched functions of each segment were then identified if passed the selection criteria. (C) The nearby segments with the same enriched function were merged to a detection window. The enrichment analysis between the function and the subset of genes in the windows were performed. The subset was constructed by excluding gene by gene along the left side or right side of the genome coordinate. The position with the smallest $p$ value of left side and right side excluding subset was defined as the boundaries of the optimal region of the functional hotspot. 

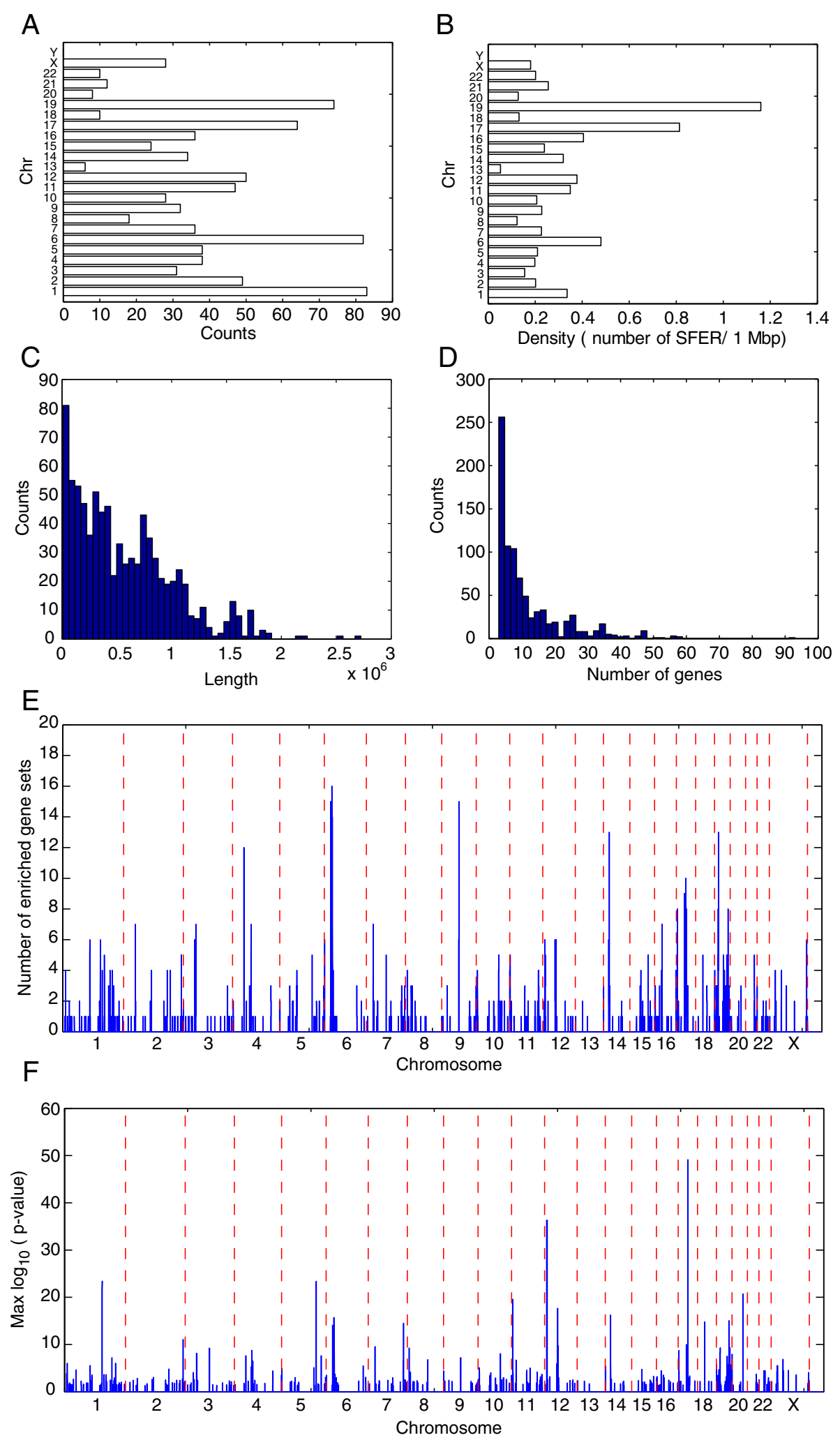

Figure 2 The summary of the spatial functional hotspots. (A) The histogram of the length of the SFHs. (B) The histogram of the gene numbers in the SFHs. (C) The SFH number in each chromosome. (D) The SFH density (numbers of SFHs per million base pair) in each chromosome. (E) The most significant $p$ value and $(\mathbf{F})$ the number of SFHs in the position of human genome. In (E) and (F), the chromosomes were separated by red broken lines. 
Gene sets of genome segments and biological functions To define the genome segments, the detection window size was set as one million base pairs (Mbp) after the testing of three different conditions (Additional file 1: Figure S1). The sliding distance was set at $0.25 \mathrm{Mbp}$. The genomic position of each gene was obtained from Ensembl (version Homo Sapiens 65) [20], or equivalent to NCBI human genome GRCh37. Therefore, a total of 12,098 segments were defined. To construct the functional gene sets, we downloaded all records of Gene Ontology from the BioMart website of Ensembl 65 (http://useast.ensembl.org/info/ data/biomart.html) [20]. A total of 7,654 GO terms were downloaded. After excluding the gene sets containing fewer than 15 genes, 1,091, 404, and 275 gene sets associated to biological process (BP), molecular function (MF), and cellular component (CC) terms were utilized in this study, respectively.

\section{aCGH arrays of hepatocellular carcinoma}

To identify the functional effect of CNAs in HCC, the aCGH array data set, GSE14322, was downloaded from GEO/NCBI website. The data set contains 76 HCC samples. The determination of CNAs was through the NEXUS software (BioDiscovery, San Diego, CA, USA). The CBS segmentation algorithm was performed to identify the segments of CNAs [21] using the thresholds of $\log 2$ values of fold change larger or smaller than \pm 0.2 .

\section{Results}

Identification of spatial functional hotspots

By using adjusted $p$ values of Fisher's exact test $<0.05$ after Bonferroni adjustment as the criteria, a total of 540 GO gene sets showed the functional enrichment in 838 SFHs. There are 443, 269, and 126 of SFHs belonging to $\mathrm{BP}, \mathrm{MF}$, and $\mathrm{CC}$ terms, respectively. On average, each chromosome contains 57 SFHs. Chromosome 1 has the largest number (147) of SFHs, and chromosome Y has no SFH (Figure 2A). The averaged SFH density is 0.43 SFHs per million base pairs (Mbp). Chromosome 6 has the highest SFH density (0.48 SFHs/Mbp) (Figure 2B). For the $838 \mathrm{SFHs}$, the average length of SFHs was 0.56 $\mathrm{Mbp}$ (Figure 2C) and the averaged 11.5 genes are in a SFH (Figure 2D). The SFH of 'sugar binding' enrichment, which is located in the 7.88 to $10.6 \mathrm{Mbp}$ region at chromosome 12, has the longest region length. The SFH of 'immune response' enrichment (31.2 to $33 \mathrm{Mbp}$ at chromosome 6), which contains 93 genes, has the largest number of genes. The region located in 29.7 to 31.5 $\mathrm{Mbp}$ at chromosome 6 contained the most number of enriched gene sets (16) (Figure 2E and Additional file 1: Table S1). The region includes lots of SFHs which have enrichment of immune-related gene sets, such like MHC class I protein complex, type I interferon-mediated signaling pathway, and immune response. Our finding indicated that the two regions are important for cell immunity.

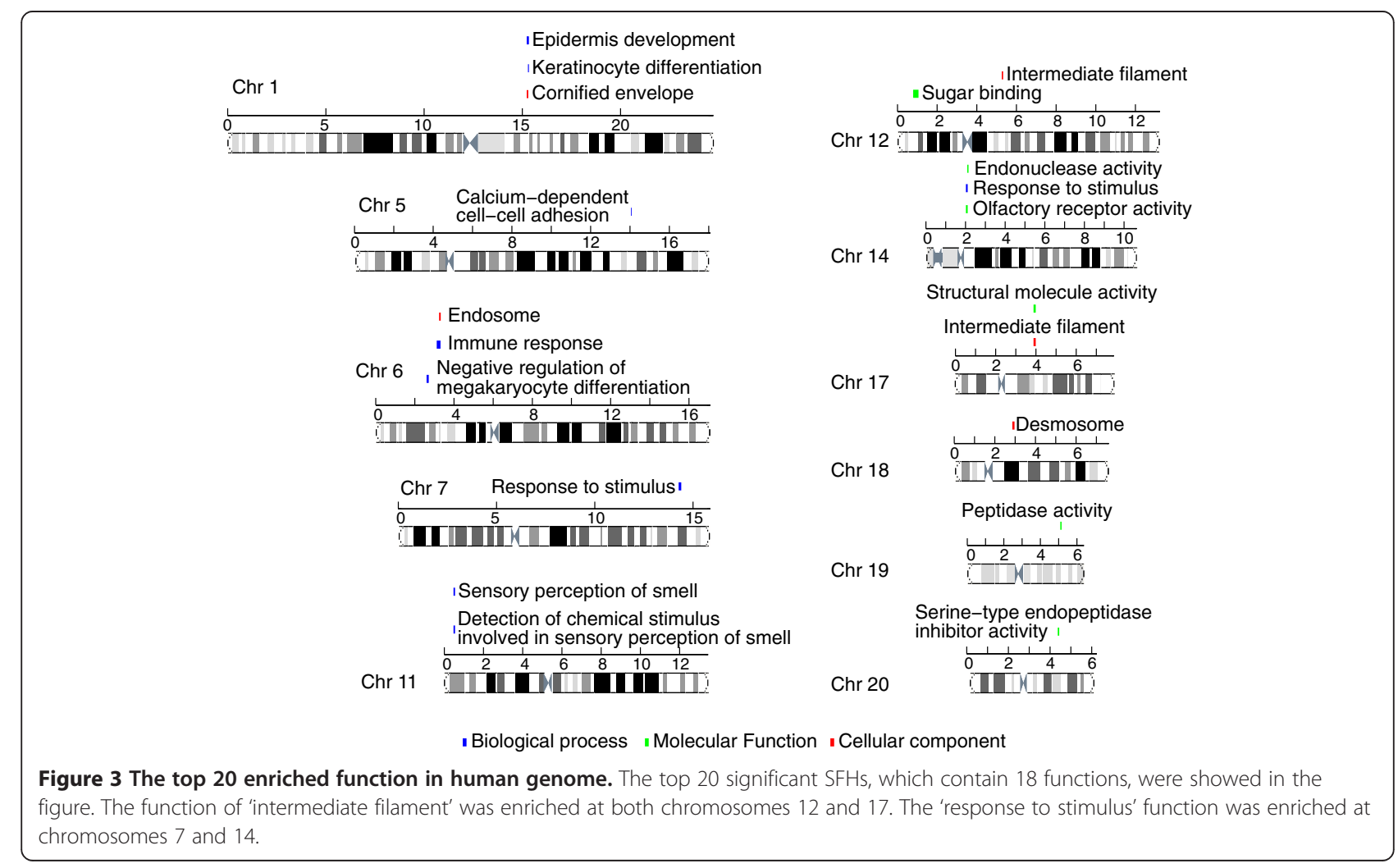


Table 1 Top ten gain/loss spatial functional hotspots (SFHs) of HCC with enriched functions

\begin{tabular}{|c|c|c|c|c|c|c|}
\hline Chr & Start pos & End pos & Number of CNVs & & Presented gene set & Number gene sets \\
\hline \multicolumn{7}{|l|}{ Gain } \\
\hline 1 & 153.33 & 153.59 & 59 & $\mathrm{bp}$ & Response to lipopolysaccharide & 4 \\
\hline 1 & 153.27 & 153.43 & 59 & $\mathrm{bp}$ & Innate immune response & 4 \\
\hline 1 & 153.33 & 153.36 & 59 & $\mathrm{bp}$ & Response to zinc ion & 2 \\
\hline 1 & 152.27 & 153.43 & 59 & $\mathrm{bp}$ & Epidermis development & 13 \\
\hline 1 & 152.27 & 153.23 & 59 & $\mathrm{mf}$ & Structural molecule activity & 8 \\
\hline 1 & 152.88 & 153.43 & 58 & $\mathrm{bp}$ & Keratinocyte differentiation & 12 \\
\hline 1 & 152.88 & 153.23 & 58 & $\mathrm{bp}$ & Peptide cross-linking & 6 \\
\hline 1 & 152.88 & 153.23 & 58 & $\mathrm{mf}$ & Protein binding, bridging & 4 \\
\hline 1 & 153.50 & 153.60 & 57 & $\mathrm{mf}$ & Protein homodimerization activity & 5 \\
\hline 1 & 153.50 & 153.60 & 57 & $\mathrm{CC}$ & Perinuclear region of cytoplasm & 5 \\
\hline \multicolumn{7}{|l|}{ Loss } \\
\hline 8 & 6.84 & 6.88 & 42 & $\mathrm{bp}$ & Response to virus & 3 \\
\hline 4 & 190.39 & 191.01 & 40 & $\mathrm{mf}$ & Sequence-specific DNA binding & 6 \\
\hline 4 & 190.39 & 191.01 & 40 & $\mathrm{mf}$ & Sequence-specific DNA binding transcription Factor activity & 6 \\
\hline 8 & 6.35 & 6.91 & 40 & $\mathrm{CC}$ & Extracellular space & 7 \\
\hline 4 & 90.80 & 91.76 & 40 & $\mathrm{CC}$ & Platelet alpha granule lumen & 2 \\
\hline 4 & 90.80 & 91.76 & 40 & $\mathrm{bp}$ & Platelet degranulation & 2 \\
\hline 8 & 26.61 & 27.47 & 39 & $\mathrm{bp}$ & Response to stress & 3 \\
\hline 17 & 10.35 & 10.56 & 38 & $\mathrm{bp}$ & Actin filament-based movement & 2 \\
\hline 8 & 26.37 & 27.31 & 37 & $\mathrm{bp}$ & Response to cocaine & 2 \\
\hline 8 & 22.88 & 23.08 & 37 & $\mathrm{mf}$ & Caspase activator activity & 2 \\
\hline
\end{tabular}

Immune-related functions are in italics.

The smallest $p$ values of the enriched gene sets in the genomic positions were showed in Figure 2F. There are nine SFHs which have adjusted $p$ value smaller than $1 \times$ $10^{-20}$. The 'intermediate filament' gene set has the most significant adjusted $p$ value $\left(7.4 \times 10^{-50}\right)$ of the enrichment in the SFH of 38.8 to $39.4 \mathrm{Mbp}$ region at chromosome 17. Twenty-nine out of 57 genes in the 'intermediate filament' gene set are located at the hotspots. All of them belong to the keratin family genes, which are components of the cytoskeleton of epithelial cells. The top 20 SFHs were showed in Figure 3. Another SFH which is located at 52.6 to $53.3 \mathrm{Mbp}$ region at chromosome 12 also showed the enrichment of intermediate filament. Other 12 keratin genes were contained. The SFH located at 31.2 to 33 Mbp regions of chromosome 6, which code 16 human leukocyte antigen (HLA) genes, enriched the immune response gene set. In summary, our result indicates that there are several functional hotspots within human genome related to the immune function.

\section{The affected function of copy number variation in liver cancer}

To evaluate the effect function of CNAs in liver cancer, the dataset GSE14322, which contains 76 aCGH arrays of HCC samples, was downloaded and analyzed. The percentage of CNA status of each SFH was calculated. There are 61 and 89 SFHs that contained copy number gain and loss in more than $30 \%$ patients (25). The result was showed in Table S2 in Additional file 1, and the top ten SFHs were listed in Table 1. One immune-related gene set had the gain status in most of the samples (innate immune response), and one had loss status (response to virus), since the major etiologies of $\mathrm{HCC}$ are the infection of $\mathrm{HBV}$ and $\mathrm{HCV}$. We hypothesize that those immune-related SFHs that harbor CNAs may play a role in the HCC carcinogenesis.

We also analyzed the association between disease-free survival and the CNAs of the SFHs through log rank test. Using $p<0.01$ as the threshold, a total of 20 and 19 SFHs of which gain and loss status were identified with survival association, respectively (see Table 2 ). The copy number gain status in the SFH which located at 41.1 to 41.9 Mbp at chromosome 19 had the smallest $p$ value of the survival testing. The SFH had the enrichment of 'oxygen binding'. As shown in Figure 4A, the patients with copy number gain in the SFH had reduced survival comparing with neutral status. Interestingly, all the SFHs with survival-associated gain status were all located at 
Table 2 The SFHs of which copy number status were associated with patient survival

\begin{tabular}{|c|c|c|c|c|c|c|}
\hline Chr & Start pos & End pos & $p$ value & & Presented gene set & Number of overlapped genes \\
\hline \multicolumn{7}{|l|}{ Gain } \\
\hline 19 & 41.38 & 41.63 & 2.6E-04 & $\mathrm{mf}$ & Oxygen binding & 2 \\
\hline 19 & 43.23 & 44.29 & 0.001 & $\mathrm{bp}$ & Defense response & 4 \\
\hline 19 & 54.72 & 55.11 & 0.002 & $\mathrm{bp}$ & Defense response & 6 \\
\hline 19 & 54.72 & 55.11 & 0.002 & $\mathrm{bp}$ & Cell surface receptor linked Signaling pathway & 6 \\
\hline 19 & 40.09 & 40.23 & 0.003 & $\mathrm{mf}$ & Lysophospholipase activity & 2 \\
\hline 19 & 40.09 & 40.23 & 0.003 & $\mathrm{mf}$ & Carboxylesterase activity & 2 \\
\hline 19 & 39.41 & 39.52 & 0.004 & $\mathrm{CC}$ & SCF ubiquitin ligase complex & 2 \\
\hline 19 & 39.41 & 39.52 & 0.004 & $\mathrm{mf}$ & Glycoprotein binding & 2 \\
\hline 19 & 51.63 & 52.15 & 0.007 & $\mathrm{mf}$ & Sugar binding & 9 \\
\hline 19 & 54.78 & 55.38 & 0.007 & $\mathrm{bp}$ & Regulation of immune response & 8 \\
\hline 19 & 51.63 & 52.27 & 0.007 & $\mathrm{bp}$ & Cell adhesion & 11 \\
\hline 19 & 42.18 & 44.32 & 0.007 & $\mathrm{CC}$ & Anchored to membrane & 9 \\
\hline 19 & 54.72 & 55.55 & 0.007 & $\mathrm{mf}$ & Transmembrane receptor activity & 8 \\
\hline 19 & 54.80 & 55.30 & 0.007 & $\mathrm{mf}$ & Antigen binding & 5 \\
\hline 19 & 54.78 & 55.42 & 0.007 & $\mathrm{bp}$ & Cellular defense response & 5 \\
\hline 19 & 54.72 & 55.55 & 0.007 & $\mathrm{CC}$ & Integral to plasma membrane & 12 \\
\hline 19 & 58.55 & 59.08 & 0.007 & $\mathrm{bp}$ & Viral reproduction & 7 \\
\hline 19 & 54.78 & 55.40 & 0.007 & $\mathrm{bp}$ & Immune response & 6 \\
\hline 19 & 50.86 & 51.59 & 0.008 & $\mathrm{mf}$ & Peptidase activity & 17 \\
\hline 19 & 45.41 & 45.45 & 0.008 & $\mathrm{mf}$ & Lipid transporter activity & 2 \\
\hline \multicolumn{7}{|l|}{ Loss } \\
\hline 8 & 11.83 & 12.18 & 2.4E-04 & $\mathrm{bp}$ & Defense response to bacterium & 4 \\
\hline 4 & 55.10 & 55.99 & 0.001 & $\mathrm{bp}$ & Vascular endothelial growth Factor receptor signaling pathway & 2 \\
\hline 4 & 55.10 & 55.99 & 0.001 & $\mathrm{mf}$ & Growth factor binding & 2 \\
\hline 8 & 22.88 & 23.08 & 0.001 & $\mathrm{mf}$ & Caspase activator activity & 2 \\
\hline 8 & 22.30 & 23.02 & 0.002 & $\mathrm{bp}$ & Apoptosis & 5 \\
\hline 4 & 74.61 & 74.97 & 0.003 & $\mathrm{bp}$ & Inflammatory response & 5 \\
\hline 8 & 26.37 & 27.47 & 0.004 & $\mathrm{CC}$ & Growth cone & 3 \\
\hline 8 & 26.37 & 27.32 & 0.004 & $\mathrm{bp}$ & Response to cocaine & 2 \\
\hline 8 & 22.01 & 23.08 & 0.005 & $\mathrm{bp}$ & Cellular response to mechanical stimulus & 3 \\
\hline 4 & 68.69 & 69.36 & 0.006 & $\mathrm{mf}$ & Serine-type endopeptidase activity & 5 \\
\hline 4 & 68.69 & 69.36 & 0.006 & $\mathrm{bp}$ & Proteolysis & 5 \\
\hline 4 & 68.69 & 69.36 & 0.006 & $\mathrm{mf}$ & Peptidase activity & 5 \\
\hline 4 & 70.86 & 71.40 & 0.007 & $\mathrm{bp}$ & Biomineral tissue development & 5 \\
\hline 4 & 71.06 & 71.47 & 0.007 & $\mathrm{bp}$ & Odontogenesis of dentine-containing tooth & 3 \\
\hline 4 & 74.26 & 74.85 & 0.007 & $\mathrm{CC}$ & Platelet alpha granule lumen & 3 \\
\hline 4 & 74.26 & 74.85 & 0.007 & $\mathrm{bp}$ & Platelet degranulation & 3 \\
\hline 4 & 76.92 & 76.94 & 0.008 & $\mathrm{bp}$ & Defense response to virus & 2 \\
\hline 4 & 74.70 & 75.32 & 0.008 & $\mathrm{bp}$ & Cell-cell signaling & 4 \\
\hline 8 & 38.13 & 38.33 & 0.008 & $\mathrm{bp}$ & Cell growth & 2 \\
\hline
\end{tabular}

chromosome 19 and ranged from $33.7 \mathrm{M}$ to $59 \mathrm{Mbp}$. Four immune-related functions, defense response, regulation of immune response, antigen binding, and immune response, were enriched in the region. The finding indicated that the immune functional island located at the region is sensitive to patient survival. The SFH located at 11.8 to 12.2 

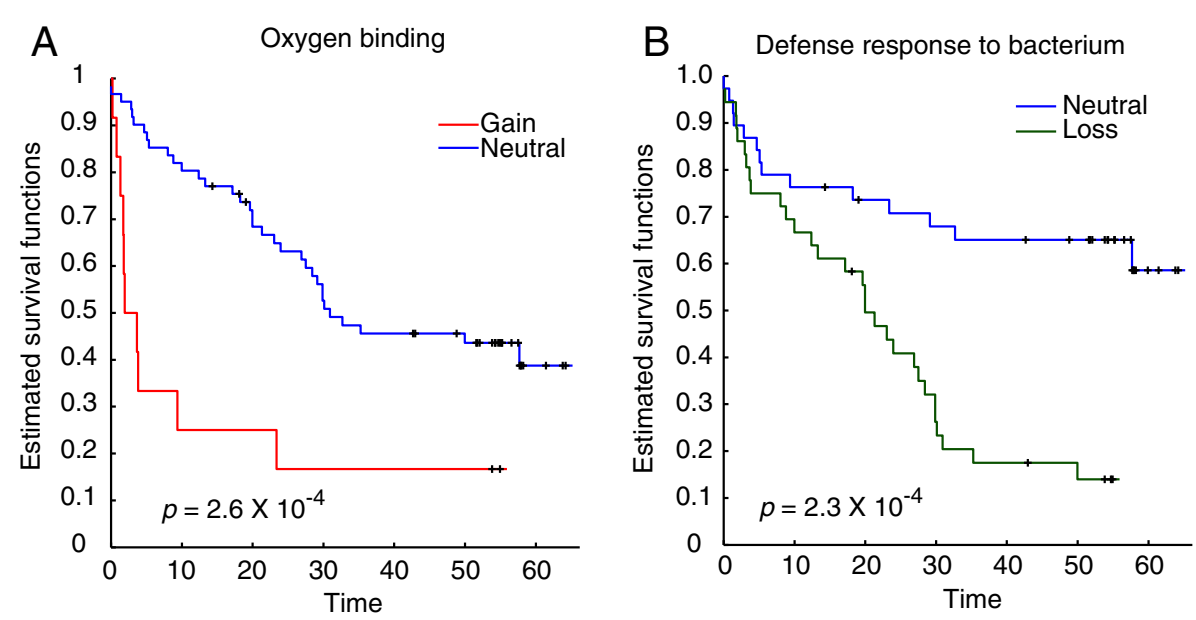

Figure 4 Patient survival correlated to copy number status of tumor. (A) Kaplan-Meier plots of patient disease-free survival were separated by the status of copy number in the SFH located at 41.1 to $41.9 \mathrm{Mbp}$ at chromosome 19. The SFH has enrichment of 'oxygen binding'. Samples were assigned to two groups, copy number gain (red line) and neutral (blue line). The $p$ values was statistically significant (<0.01). (B) KaplanMeier plots of the copy number status in SFH at 11.8 to $12.2 \mathrm{Mbp}$ at chromosome 8. Samples were assigned to two groups, copy number loss (green line) and neutral (blue line). The SFH had enrichment of 'defense response to bacterium'. The $p$ value of test was also significant.

Mbp at chromosome 8, which has enrichment of 'defense response to bacterium, has the smallest $p$ value of copy number loss status (Figure 4B). For SFHs with survivalassociated loss status, 12 of them were located at 55 to $76.9 \mathrm{Mbp}$ region at chromosome 4 , and 7 of them were located at 11.1 to $38.3 \mathrm{Mbp}$ at chromosome 8 .

\section{Discussion}

We introduced a system biology method, motivated by Cancer Gene Island, to identify the spatial functional hotspots in human genome. A statistical assay was presented to estimate the enrichment within genome regions to functional gene sets. By applying the terms of Gene Ontology into our method, the result provided the details of the function encoded in human genome. We set the two parameters of the algorithm, the length of window size and shift distance, as 1 and $0.25 \mathrm{Mbp}$, respectively. Although the setting of the parameters will affect the $p$ value of enrichment testing for each segment, our algorithm performed an optimal procedure which merge the continual enriched segments and find the region with maximum $p$ values by removing the gene one by one from both sides. Different settings of window size will not affect the results of final optimal regions. However, the detection of continual functional enriched segments could be missed under the condition of small window size because the windows contained no and less genes. To find out the workable parameters, we tested three conditions of window sizes, $0.5,1$, and 1.5 $\mathrm{Mbp}$ and found out that the condition of $0.5 \mathrm{Mbp}$ contains large numbers of segments of which the gene number is less than three. The parameters of 1 and $1.5 \mathrm{Mbp}$ contain fewer segments with low numbers of genes. Through the testing, we set the window size as $1 \mathrm{Mbp}$ to analyze the human genome.

We applied the method in HCC data set to estimate the effect of hotspots in the genome. Using the data set GSE14322 as an example, a total of 150 SFHs have been identified with copy number alterations in most of the HCC patients, and the novelty of our analysis is to identify the functional hotspots in human genome. The region we identified is located with high density of genes that share the same biological function, and as we demonstrated in the HCC dataset, these functions may also be sensitive to CNAs. Two immune-related functional regions were identified with gain or loss in most of patients in the HCC dataset. The major carcinogenesis of $\mathrm{HCC}$ is the chronic and acute inflammation under HBV or HCV infection; thus, we hypothesize that these two regions we identified may also play a role in $\mathrm{HCC}$ oncogenesis.

We also identified 39 SFHs of which the copy number status was associated with patient survival. The result indicates that the copy number alterations in these regions may affect the function of tumor progression and then reflect on patient survival. For example, the patients who have copy number loss in the SFH which was enriched in inflammatory response have shorter survival. The chronic and acute inflammations induced by HBV and HCV infection have been proved to play an important role in HCC tumorgenesis [22,23]. Our data implied that copy number alterations may contribute in the inflammatory response. Also, other enriched functions in survival-related SFHs have been reported, such as 
regulation of immune response, cell growth, apoptosis, and caspase activator activity. The SFHs and enriched function we identified provided the clues of the association between CNAs and the regulations of the enriched functions. We expected that the SFHs we identified will provide further insight of affected functions of CNAs to uncover the mechanism of cancer.

\section{Conclusions}

In this paper, we systematically surveyed human genome and identified 838 functional hotspots based on Gene Ontology classification. To substantiate our findings, 76 HCC tumors and their DNA copy number gain/loss statuses were examined closely. Among the 838 hotspots, a total of 150 regions affected by CNAs, and the encoded enriched functions were identified. Our results indicate that two immune-related hotspots had copy number alterations in most of the patients and might be involved in HCC oncogenesis. In addition, 39 survival-related hotspots were identified. Taken together, our results demonstrated that the method presented in the paper is a powerful tool to survey biological functions of CNAs and to construct regulation hypothesis at pathway and functional levels.

\section{Additional file}

Additional file 1: Supplemental materials. This file contains tables and a figure showing the enriched functions, spatial functional hotspots, and histogram of the gene numbers.

\section{Competing interests}

All authors declare that they have no competing interests.

\section{Acknowledgements}

This work was supported by the NIH/NCl Cancer Center grant (P30 CA054174-17), NSF grant (NSF CCF-1246073), and Qatar National Research Foundation (NPRPO9 -874-3-235). TH is supported by NIH/NCl grant (U54 CA113001) and the Greehey Children Cancer Research Institute (GCCRI) Intramural Research fund. SR and XWW were supported by grants (Z01-BC 010313) from the Intramural Research Program of the Center for Cancer Research, the National Cancer Institute. The funders had no role in the study design, data collection and analysis, decision to publish, or preparation of the manuscript.

\section{Author details \\ ${ }^{1}$ Greehey Children's Cancer Research Institute, University of Texas Health Science Center at San Antonio, San Antonio, TX 78229, USA. ${ }^{2}$ Institute of Pathology, University Hospital, Im Neuenheimer Feld 224, Room 2.034, Heidelberg 69120, Germany. ${ }^{3}$ Laboratory of Human Carcinogenesis, National Cancer Institute, NIH, Bethesda, MD 20892, USA. ${ }^{4}$ Department of Epidemiology and Biostatistics, University of Texas Health Science Center at San Antonio, San Antonio, TX 78229, USA.}

\section{Received: 31 May 2013 Accepted: 8 October 2013}

Published: 25 October 2013

\section{References}

1. SL Carter, AC Eklund, IS Kohane, LN Harris, Z Szallasi, A signature of chromosomal instability inferred from gene expression profiles predicts clinical outcome in multiple human cancers. Nat. Genet. 38, 1043-1048 (2006)
2. HG Russnes, HK Vollan, OC Lingjaerde, A Krasnitz, P Lundin, B Naume, T Sørlie, E Borgen, IH Rye, A Langerød, SF Chin, AE Teschendorff, PJ Stephens, S Månér, E Schlichting, LO Baumbusch, R Kåresen, MP Stratton, M Wigler, C Caldas, A Zetterberg, J Hicks, AL Børresen-Dale, Genomic architecture characterizes tumor progression paths and fate in breast cancer patients. Sci. Transl. Med 2, 38ra47 (2010)

3. P Micke, K Edlund, L Holmberg, HG Kultima, L Mansouri, S Ekman, M Bergqvist, L Scheibenflug, K Lamberg, G Myrdal, A Berglund, A Andersson, M Lambe, F Nyberg, A Thomas, A Isaksson, J Botling, Gene copy number aberrations are associated with survival in histologic subgroups of non-small cell lung cancer. J. Thorac. Oncol. 6, 1833-1840 (2011)

4. K Kurashina, Y Yamashita, T Ueno, K Koinuma, J Ohashi, H Horie, Y Miyakura, T Hamada, H Haruta, H Hatanaka, M Soda, YL Choi, S Takada, Y Yasuda, H Nagai, H Mano, Chromosome copy number analysis in screening for prognosis-related genomic regions in colorectal carcinoma. Cancer Sci. 99, 1835-1840 (2008)

5. M Tan, D Yu, Molecular mechanisms of erbB2-mediated breast cancer chemoresistance. Adv. Exp. Med. Biol. 608, 119-129 (2007)

6. JE Lucas, HN Kung, JT Chi, Latent factor analysis to discover pathwayassociated putative segmental aneuploidies in human cancers. PLoS Comput. Biol. 6, e1000920 (2010)

7. NL Solimini, Q Xu, CH Mermel, AC Liang, MR Schlabach, J Luo, AE Burrows, AN Anselmo, AL Bredemeyer, MZ Li, R Beroukhim, M Meyerson, SJ Elledge, Recurrent hemizygous deletions in cancers may optimize proliferative potential. Science 337, 104-109 (2012)

8. X Tang, JE Lucas, JL Chen, G LaMonte, J Wu, MC Wang, C Koumenis, JT Chi, Functional interaction between responses to lactic acidosis and hypoxia regulates genomic transcriptional outputs. Cancer Res. 72, 491-502 (2012)

9. W da Huang, BT Sherman, RA Lempicki, Bioinformatics enrichment tools: paths toward the comprehensive functional analysis of large gene lists. Nucleic Acids Res 37, 1-13 (2009)

10. SA Tomlins, R Mehra, DR Rhodes, X Cao, L Wang, SM Dhanasekaran, S Kalyana-Sundaram, JT Wei, MA Rubin, KJ Pienta, RB Shah, AM Chinnaiyan, Integrative molecular concept modeling of prostate cancer progression. Nat. Genet. 39, 41-51 (2007)

11. Q Zheng, XJ Wang, GOEAST: a web-based software toolkit for Gene Ontology enrichment analysis. Nucleic Acids Res 36, W358-W363 (2008)

12. W da Huang, BT Sherman, RA Lempicki, Systematic and integrative analysis of large gene lists using DAVID bioinformatics resources. Nat. Protoc. 4, 44-57 (2009)

13. DM Parkin, F Bray, J Ferlay, P Pisani, Global cancer statistics, 2002. CA. Cancer. J. Clin. 55, 74-108 (2005)

14. PA Farazi, RA DePinho, Hepatocellular carcinoma pathogenesis: from genes to environment. Nat. Rev. Cancer 6, 674-687 (2006)

15. C Schlaeger, T Longerich, C Schiller, P Bewerunge, A Mehrabi, G Toedt, J Kleeff, V Ehemann, R Eils, P Lichter, P Schirmacher, B Radlwimmer, Etiologydependent molecular mechanisms in human hepatocarcinogenesis. Hepatology 47, 511-520 (Feb 2008)

16. MA Patil, I Gutgemann, J Zhang, C Ho, ST Cheung, D Ginzinger, R Li, KJ Dykema, S So, ST Fan, S Kakar, KA Furge, R Buttner, X Chen, Array-based comparative genomic hybridization reveals recurrent chromosomal aberrations and Jab1 as a potential target for 8q gain in hepatocellular carcinoma. Carcinogenesis 26, 2050-2057 (2005)

17. L Zender, MS Spector, W Xue, P Flemming, C Cordon-Cardo, J Silke, ST Fan, JM Luk, M Wigler, GJ Hannon, D Mu, R Lucito, S Powers, SW Lowe, Identification and validation of oncogenes in liver cancer using an integrative oncogenomic approach. Cell 125, 1253-1267 (2006)

18. L Zender, W Xue, J Zuber, CP Semighini, A Krasnitz, B Ma, P Zender, S Kubicka, JM Luk, P Schirmacher, WR McCombie, M Wigler, J Hicks, GJ Hannon, S Powers, SW Lowe, An oncogenomics-based in vivo RNAi screen identifies tumor suppressors in liver cancer. Cell 135, 852-864 (2008)

19. S Roessler, EL Long, A Budhu, Y Chen, X Zhao, J Ji, R Walker, HL Jia, QH Ye, LX Qin, ZY Tang, P He, KW Hunter, SS Thorgeirsson, PS Meltzer, XW Wang, Integrative genomic identification of genes on $8 p$ associated with hepatocellular carcinoma progression and patient survival. Gastroenterology 142, 957-966 (2012)

20. P Flicek, MR Amode, D Barrell, K Beal, S Brent, D Carvalho-Silva, P Clapham, G Coates, S Fairley, S Fitzgerald, L Gil, L Gordon, M Hendrix, T Hourlier, N Johnson, AK Kähäri, D Keefe, S Keenan, R Kinsella, M Komorowska, G Koscielny, E Kulesha, P Larsson, I Longden, W McLaren, M Muffato, B Overduin, M Pignatelli, B Pritchard, HS Riat, Ensembl 2012. Nucleic Acid Res 40, D84-D90 (2012) 
21. AB Olshen, ES Venkatraman, R Lucito, M Wigler, Circular binary segmentation for the analysis of array-based DNA copy number data. Biostatistics 5, 557-572 (2004)

22. M Levrero, Viral hepatitis and liver cancer: the case of hepatitis C. Oncogene 25, 3834-3847 (2006)

23. D Kremsdorf, P Soussan, P Paterlini-Brechot, C Brechot, Hepatitis B virusrelated hepatocellular carcinoma: paradigms for viral-related human carcinogenesis. Oncogene 25, 3823-3833 (2006)

doi:10.1186/1687-4153-2013-14

Cite this article as: Hsiao et al:: Identification of genomic functional

hotspots with copy number alteration in liver cancer. EURASIP Journal on

Bioinformatics and Systems Biology 2013 2013:14.

Submit your manuscript to a SpringerOpen ${ }^{\circ}$ journal and benefit from:

- Convenient online submission

- Rigorous peer review

- Immediate publication on acceptance

- Open access: articles freely available online

- High visibility within the field

- Retaining the copyright to your article

Submit your next manuscript at $\gg$ springeropen.com 